\title{
Coercion-Resistance and Receipt-Freeness in Electronic Voting *
}

\author{
Stéphanie Delaune \\ LSV, France Télécom R\&D \\ ENS Cachan, CNRS, France \\ delaune@1sv.ens-cachan.fr
}

\author{
Steve Kremer \\ LSV, INRIA \\ ENS Cachan, CNRS, France \\ kremer@1sv.ens-cachan.fr
}

\author{
Mark Ryan \\ School of Computer Science \\ University of Birmingham, UK \\ M.D.Ryan@cs.bham.ac.uk
}

\begin{abstract}
In this paper we formally study important properties of electronic voting protocols. In particular we are interested in coercion-resistance and receipt-freeness. Intuitively, an election protocol is coercion-resistant if a voter A cannot prove to a potential coercer $C$ that she voted in a particular way. We assume that $A$ cooperates with $C$ in an interactive fashion. Receipt-freeness is a weaker property, for which we assume that $A$ and $C$ cannot interact during the protocol: to break receipt-freeness, A later provides evidence (the receipt) of how she voted. While receipt-freeness can be expressed using observational equivalence from the applied pi calculus, we need to introduce a new relation to capture coercion-resistance. Ourformalization of coercionresistance and receipt-freeness are quite different. Nevertheless, we show in accordance with intuition that coercionresistance implies receipt-freeness, which implies privacy, the basic anonymity property of voting protocols, as de£ned in previous work. Finally we illustrate the de£nitions on a simplifed version of the Lee et al. voting protocol.
\end{abstract}

\section{Introduction}

Electronic voting promises the possibility of a convenient, effcient and secure facility for recording and tallying votes. Many protocols specifying the interaction between voters and election administrators have been proposed $[6,10,12,3,11,7,13,15]$. While voting of this kind appears to encourage higher voter turnout, it also carries the potential of making abuse easier to perform and easier to perform at a large scale. Such protocols involve a high level of concurrency and even small protocols are known to be extremely error-prone. Formal analysis is crucial to assess their security.

*19th IEEE Computer Security Foundations Workshop (CSFW), 2006. This work has been partly supported by the RNTL project PROUVÉ 03V360 and the ACI-SI Rossignol.
Among the properties considered desirable of electronic voting protocols are the following:

Privacy: the system cannot reveal how a particular voter voted.

Coercion-resistance: a voter cannot cooperate with a coercer to prove to him that she voted in a certain way.

The privacy property guarantees that the link between a voter and her vote remains secret. Coercion-resistance guarantees that the coercer cannot become convinced of how a voter votes, even if the voter cooperates with him. Of course the voter can tell a coercer how she voted, but coercionresistance asserts that she is unable to prove it, so the coercer has no reason to believe her. Intuitively, coercionresistance is a stronger property than privacy, since if it is possible for a coercer to detect the value of a voter's vote without the voter's cooperation, then it is certainly also possible with the voter's cooperation. Receipt-freeness is a related property that has also been studied in the literature, which is between coercion-resistance and privacy in strength:

Receipt-freeness: a voter does not gain any information (a receipt) which can be used to prove to a coercer that she voted in a certain way.

Note that in literature the distinction between receiptfreeness and coercion-resistance is not very clear. The definitions are usually given in natural language and are insuf£ciently precise to allow comparison. The notion of receipt-freeness frst appeared in [4]. Since then, several schemes $[4,16]$ were proposed in order to meet the condition of receipt-freeness, but later shown not to satisfy it. One of the reasons for such paws is that no formal defnition of receipt-freeness has been given. The situation for coercion-resistance is similar. Systems have been proposed aiming to satisfy it; for example, Okamoto [17] presents a system resistant to interactive coercers, thus aiming to satisfy what we call coercion-resistance, but this property is stated only in natural language. Recently, a rigorous definition in a computational model has been proposed for 
coercion-resistance [13]. We present in this paper what we believe to be the £rst "formal methods" defnition of receipt-freeness and coercion-resistance. We use the applied pi calculus framework and we make a clear distinction between the two notions. One of the advantages of the applied pi calculus is that it allows us to reason about equational theories that allow us to model the less classical cryptographic primitives often used in voting protocols. It is diffcult to compare our defnition and the one given in [13] due to the inherently different models.

As is often done in protocol analysis, we assume the Dolev-Yao abstraction: cryptographic primitives are assumed to work perfectly, and the attacker controls the public channels. The attacker can see, intercept and insert messages on a public channel, but can only encrypt, decrypt or sign messages for which he has the relevant key. In the case of both receipt-freeness and coercion-resistance, we assume that the coercer has all the capabilities of the attacker on the public channels.

We consider that the difference between receipt-freeness and coercion-resistance lies in the powers of the coercer to interact with the voter during the voting stage. In receiptfreeness, we assume a coercer who simply examines evidence gained from observing the election process. Such evidence includes information provided by the cooperating voter, e.g., the voter's private key and random coins used for probabilistic encryption. In coercion-resistance, the coercer has additional capabilities. He can interact with the cooperating voter, for example by (adaptively) preparing messages which the voter will send during the process.

Coercion-resistance cannot possibly hold if the coercer can physically vote on behalf of the voter. Some mechanism is necessary for isolating the voter from the coercer at the moment she casts her vote. This can be realised by a voting booth, which we model here as a private and anonymous channel between the voter and the election administrators.

Receipt-freeness is formalised as an observational equivalence. Intuitively, a protocol is receipt-free if a coercer cannot detect a difference between Alice voting in the way he instructed, and her voting in some other way, provided Bob votes in the complementary way each time. (The purpose of introducing Bob here is to prevent the observer seeing a different number of votes for each candidate.) Alice cooperates with the coercer by sharing secrets, but the coercer cannot interact with Alice to give her some prepared messages.

In the case of coercion-resistance, the coercer is assumed to communicate with Alice during the protocol, and can prepare messages which she should send during the election process. This gives the coercer much more power. It turns out that observational equivalence is not dexible enough, and we generalise it to a notion which we call adaptive simulation. We expect that adaptive simulation will prove to be of interest for other kinds of properties and protocols than the ones we study here.

Although the three properties are formalised in markedly different ways, we prove that, in accordance with intuition, coercion-resistance implies receipt-freeness, which in turn implies privacy.

It is rather classical to formalize anonymity properties as some kind of observational equivalence in a process algebra or calculus, going back to the work of Schneider and Sidiropoulos [18]. However, to the best of our knowledge this is the Erst formalisation of the notion of not being able to prove in this kind of framework.

Our formalization of coercion-resistance gives interesting insights, such as the ability of the coercer to perform fault attacks. This kind of attack is also mentioned in [13] as a "randomization attack". The idea of a fault attack is to let the coercer test the behavior of a coerced voter by requiring this voter to send a garbage message at some point of the protocol. If the voter is unable to decide whether the message is garbage or not, the attacker may distinguish a voter following the coercer's instructions from a voter who is trying to cheat the coercer, as the protocol would block on the incorrect message. Verifying whether a message is correct or not is often diffcult when for instance ciphertexts are sent. In practice, a coercer can use this technique to probabilistically check whether a coerced voter is behaving as expected. Avoiding these attacks requires to carefully choose some of the implementation issues.

Finally, we illustrate our defnitions and the idea of a fault attack on a simplifed version of a voting protocol proposed by Lee $e t$ al. [15]. Due to lack of space, some proofs are omitted. They can be found in [8].

\section{The applied pi calculus}

The applied pi calculus [2] is a language for describing concurrent processes and their interactions. It is based on the pi calculus, but is intended to be less pure and therefore more convenient to use. The applied pi calculus has been used to study a variety of security protocols, such as those for private authentication [9], for key establishment [1], as well as an electronic voting protocol [14].

To describe processes in the applied pi calculus, one starts with a set of names (which are used to name communication channels or other constants), a set of variables, and a signature $\Sigma$ which consists of the function symbols which will be used to defne terms. In the case of security protocols, typical function symbols will include enc for encryption, which takes plaintext and a key and returns the corresponding cipher text, and dec for decryption, taking ciphertext and a key and returning the plaintext. Terms are defned as names, variables, and function symbols applied to other terms. Terms and function 
symbols are sorted, and of course function symbol application must respect sorts and arities. By the means of an equational theory $E$ we describe the equations which hold on terms constructed from the signature. We denote $=_{E}$ the equivalence relation induced by $E$. A typical example of an equational theory useful for cryptographic protocols is $\operatorname{dec}(\operatorname{enc}(x, k), k)=x$. For example, given this theory and terms $T_{1}=\operatorname{dec}\left(\operatorname{enc}\left(\operatorname{enc}\left(n, k_{1}\right), k_{2}\right), k_{2}\right)$ and $T_{2}=\operatorname{enc}\left(n, k_{1}\right)$, we have $T_{1}={ }_{E} T_{2}$ (while obviously the syntactic equality $T_{1}=T_{2}$ does not hold). We write $\operatorname{vars}(T)$ for the set of variables occuring in $T$. When $\operatorname{vars}(T)=\emptyset$ we say that the term $T$ is ground.

In the applied pi calculus, one has (plain) processes and extended processes. Plain processes are built up in a similar way to processes in the pi calculus, except that messages can contain terms (rather than just names). In the grammar described below, $M$ and $N$ are terms, $n$ is a name, $x$ a variable and $u$ is a metavariable, standing either for a name or a variable.

$$
\begin{array}{cc}
P, Q, R:= & \text { plain processes } \\
0 & \text { null process } \\
P \mid Q & \text { parallel composition } \\
! P & \text { replication } \\
\nu n . P & \text { name restriction } \\
\text { if } M=N \text { then } P \text { else } Q & \text { conditional } \\
\text { in }(u, x) . P & \text { message input } \\
\text { out }(u, N) . P & \text { message output }
\end{array}
$$

Extended processes add active substitutions and restriction on variables:

$$
\begin{aligned}
& A, B, C:= \\
& P \\
& A \mid B \\
& \nu n . A \\
& \nu x . A \\
&\left\{{ }^{M} / x\right\}
\end{aligned}
$$

$$
\begin{aligned}
& \text { extended processes } \\
& \text { plain process } \\
& \text { parallel composition } \\
& \text { name restriction } \\
& \text { variable restriction } \\
& \text { active substitution }
\end{aligned}
$$

$\left\{{ }^{M} / x\right\}$ is the substitution that replaces the variable $x$ with the term $M$. Active substitutions generalise "let". The process $\nu x .\left(\left\{{ }^{M} / x\right\} \mid P\right)$ corresponds exactly to "let $x=M$ in $P$ '. As usual, names and variables have scopes, which are delimited by restrictions and by inputs. We write $f v(A)$, $b v(A), f n(A)$ and $b n(A)$ for the sets of free and bound variables and free and bound names of $A$, respectively. We say that an extended process is closed if all its variables are either bound or defned by an active substitution.

Active substitutions are useful because they allow us to map an extended process $A$ to its frame $\phi(A)$ by replacing every plain process in $A$ with 0 . A frame is an extended process built up from 0 and active substitutions by parallel composition and restriction. The frame $\phi(A)$ can be viewed as an approximation of $A$ that accounts for the static knowledge $A$ exposes to its environment, but not $A$ 's dynamic behaviour. The domain of a frame $\varphi, \operatorname{dom}(\varphi)$, is the set of variables for which $\varphi$ de£nes a substitution (those variables $x$ for which $\varphi$ contains a substitution $\{M / x\}$ not under a restriction on $x$ ). We denote by $\sigma_{\mid X}$ the substitution $\sigma$ restricted to the set of variables $X$, i.e., $\sigma_{\mid X}=\sigma(x)$ if $x \in X$ and $\sigma_{\mid X}=x$ otherwise.

An evaluation context $\left.C_{[-}\right]$is an extended process with a hole instead of an extended process. Structural equivalence, noted $\equiv$, is the smallest equivalence relation on extended processes that is closed under $\alpha$-conversion on names and variables, by application of evaluation contexts, and satisfying some further basic structural rules such as $A \mid 0 \equiv A$, associativity and commutativity of $\mid$, binding-operator-like behaviour of $\nu$, and when $M={ }_{E} N$ the equivalences

$$
\begin{gathered}
\nu x \cdot\left\{{ }^{M} / x\right\} \equiv 0 \quad\left\{{ }^{M} / x\right\} \equiv\left\{{ }^{N} / x\right\} \\
\left\{{ }^{M} / x\right\}\left|A \equiv\left\{{ }^{M} / x\right\}\right| A\left\{{ }^{M} / x\right\} .
\end{gathered}
$$

Example 1 Consider the following process $P$ :

$$
\nu s, k .\left(\operatorname{out}\left(c_{1}, \operatorname{enc}(s, k)\right) \mid \operatorname{in}\left(c_{1}, y\right) . \operatorname{out}\left(c_{2}, \operatorname{dec}(y, k)\right)\right) \text {. }
$$

The £rst component publishes the message enc $(s, k)$ by sending it on $c_{1}$. The second receives a message on $c_{1}$, uses the secret key $k$ to decrypt it, and forwards the resulting plaintext on $c_{2}$. $P$ is structurally equivalent to the following extended process $A$ :

$$
\begin{gathered}
A=\nu s, k, x_{1}, x_{2} \cdot\left(\operatorname{out}\left(c_{1}, x_{1}\right)\left|\operatorname{in}\left(c_{1}, y\right) \cdot \operatorname{out}\left(c_{2}, x_{2}\right)\right|\right. \\
\left.\left\{\operatorname{enc}(s, k) / x_{1}, \operatorname{dec}(y, k) / x_{2}\right\}\right)
\end{gathered}
$$

We have $\phi(A)=\nu s, k, x_{1}, x_{2} \cdot\left\{e n c(s, k) / x_{1}, k / x_{2}\right\} \equiv 0$ ( $x_{1}$ and $x_{2}$ are under a restriction).

The following lemma will be useful in the remaining of the paper.

Lemma 1 Let $C_{1}, C_{2}$ be two evaluation contexts. For any extended process $A$, we have $C_{1}\left[C_{2}[A]\right] \equiv C_{2}\left[C_{1}[A]\right]$.

We can now defne what it means for two frames to be statically equivalent [2].

De£nition 1 (Static equivalence) Two closed frames $\varphi_{1}$ and $\varphi_{2}$ are statically equivalent, written $\varphi_{1} \approx_{s} \varphi_{2}$, if and only if, for some names $\tilde{n}_{1}, \tilde{n}_{2}$ and substitutions $\sigma_{1}, \sigma_{2}$, such that $\varphi_{1} \equiv \nu \tilde{n}_{1} \sigma_{1}$ and $\varphi_{2} \equiv \nu \tilde{n}_{2} \sigma_{2}$, we have

(i) $\operatorname{dom}\left(\varphi_{1}\right)=\operatorname{dom}\left(\varphi_{2}\right)$,

(ii) for all terms $M, N$ with variables included in $\operatorname{dom}\left(\varphi_{i}\right)$ and using no names occurring in $\tilde{n}_{1}$ or $\tilde{n}_{2}, M \sigma_{1}={ }_{E}$ $N \sigma_{1}$ is equivalent to $M \sigma_{2}={ }_{E} N \sigma_{2}$. 
Two extended processes $A$ and $B$ are statically equivalent if and only if $\phi(A) \approx_{s} \phi(B)$.

Example 2 Let $\varphi_{0}=\nu k \cdot \sigma_{0}$ and $\varphi_{1}=\nu k \cdot \sigma_{1}$ where $\sigma_{0}=\left\{\operatorname{enc}\left(s_{0}, k\right) / x_{1}, k / x_{2}\right\}, \sigma_{1}=\left\{e n c\left(s_{1}, k\right) / x_{1}, k / x_{2}\right\}$ and $s_{0}, s_{1}$ and $k$ are names. Let $E$ be the theory defned by the axiom $\operatorname{dec}(\operatorname{enc}(x, k), k)=x$. We have $\operatorname{dec}\left(x_{1}, x_{2}\right) \sigma_{0}={ }_{E} s_{0}$ but not $\operatorname{dec}\left(x_{1}, x_{2}\right) \sigma_{1}={ }_{E} s_{0}$. Therefore, $\varphi_{0} \not_{s} \varphi_{1}$ although we have

$$
\nu k .\left\{\operatorname{enc}\left(s_{0}, k\right) / x_{1}\right\} \approx_{s} \nu k \cdot\left\{\operatorname{enc}\left(s_{1}, k\right) / x_{1}\right\} .
$$

The operational semantics of processes in the applied pi calculus is defned by structural rules defning two relations: structural equivalence (described above) and internal reduction, noted $\rightarrow$. Internal reduction $\rightarrow$ is the smallest relation on extended processes closed under structural equivalence and application of evaluation contexts such that $\operatorname{out}(a, x) . P|\operatorname{in}(a, x) \cdot Q \rightarrow P| Q$ and for any ground terms $M$ and $N$, whenever $M \neq_{E} N$, we have

$$
\begin{aligned}
& \text { if } M=M \text { then } P \text { else } Q \rightarrow P \\
& \text { if } M=N \text { then } P \text { else } Q \rightarrow Q \text {. }
\end{aligned}
$$

The operational semantics is extended by a labeled operational semantics enabling us to reason about processes that interact with their environment. Labeled operational semantics defnes the relation $\stackrel{\alpha}{\rightarrow}$ where $\alpha$ is either an input, or the output of a channel name or a variable of base type. We adopt the following rules in addition to the internal reduction rules.

$$
\begin{aligned}
& \operatorname{in}(a, x) . P \stackrel{i n(a, M)}{\longrightarrow} P\left\{{ }^{M} / x\right\} \\
& \operatorname{out}(a, u) . P \stackrel{\text { out }(a, u)}{\longrightarrow} P \\
& \frac{A \stackrel{\text { out }(a, u)}{\longrightarrow} A^{\prime} \quad u \neq a}{\nu u . A \stackrel{\text { Lu.out }(a, u)}{\longrightarrow} A^{\prime}} \\
& \frac{A \stackrel{\alpha}{\rightarrow} A^{\prime} \quad u \text { does not occur in } \alpha}{\nu u . A \stackrel{\alpha}{\longrightarrow} \nu u . A^{\prime}} \\
& \frac{A \stackrel{\alpha}{\rightarrow} A^{\prime} \quad b v(\alpha) \cap f v(B)=b n(\alpha) \cap f n(B)=\emptyset}{A\left|B \stackrel{\alpha}{\longrightarrow} A^{\prime}\right| B} \\
& \frac{A \equiv B \quad B \stackrel{\alpha}{\rightarrow} B^{\prime} \quad A^{\prime} \equiv B^{\prime}}{A \stackrel{\alpha}{\rightarrow} A^{\prime}}
\end{aligned}
$$

Note that the labeled transition is not closed under application of evaluation contexts. Moreover the output of a term $M$ needs to be made "by reference" using a restricted variable and an active substitution.

Defnition 2 (Labeled bisimilarity $\left(\approx_{\ell}\right)$ ) Labeled bisimilarity is the largest symmetric relation $\mathcal{R}$ on closed extended processes, such that $A \mathcal{R} B$ implies

$$
\text { 1. } A \approx_{s} B \text {, }
$$

2. if $A \rightarrow A^{\prime}$, then $B \rightarrow^{*} B^{\prime}$ and $A^{\prime} \mathcal{R} B^{\prime}$ for some $B^{\prime}$,

3. if $A \stackrel{\alpha}{\rightarrow} A^{\prime}$, then $B \rightarrow^{*} \stackrel{\alpha}{\rightarrow} \rightarrow * B^{\prime}$ and $A^{\prime} \mathcal{R} B^{\prime}$ for some $B^{\prime}$.

In [2], it is shown that labeled bisimilarity coincides with observational equivalence. We prefer to work with labeled bisimilarity, rather than observational equivalence, because proofs for labeled bisimilarity are generally easier. Labeled bisimilarity can be used to formalize many security properties, in particular anonymity properties, such as those studied in this paper.

\section{Formalisation}

In this section, we show how both receipt-freeness and coercion-resistance can be formalised. While receiptfreeness is expressed in terms of labeled bisimilarity, coercion-resistance requires the defnition of a new simulation relation which we call adaptive simulation.

\subsection{Voting protocols in applied pi calculus}

Before defning the properties, we need to defne what is an electronic voting protocol in applied pi calculus. Different voting protocols often have substantial differences. However, we believe that a large class of voting protocols can be represented by processes corresponding to the following structure.

Defnition 3 (Voting process) A voting process is a closed plain process

$$
V P \equiv \nu \tilde{n} .\left(V \sigma_{1}|\cdots| V \sigma_{n}\left|A_{1}\right| \cdots \mid A_{m}\right) .
$$

The $V \sigma_{i}$ are the voter processes, the $A_{j}$ s the different election authorities and the $\tilde{n}$ are channel names. We also suppose that $v \in \operatorname{dom}\left(\sigma_{i}\right)$ is a variable which refers to the value of the vote and at some moment the outcome of the vote is made public. More formally, this means that there exists A such that:

- $V P\left(\rightarrow^{*} \stackrel{\alpha}{\rightarrow}\right)^{*} A$, and

- $\phi(A) \equiv \varphi \mid\left\{v \sigma_{1} / x_{1}, \ldots,{ }^{v \sigma_{n}} / x_{n}\right\}$ for some $\varphi$.

We also defne an evaluation context $S$ which is as VP, but has a hole instead of two of the $V \sigma_{i}$.

Note that it is likely that a voting process will have the property that for any permutation $\pi$ on $\{1, \ldots, n\}$ there exists a process $A$ such that $V P\left(\rightarrow^{*} \stackrel{\alpha}{\rightarrow}\right)^{*} A$ and $\phi(A) \equiv \varphi \mid$ $\left\{v \sigma_{1} / x_{\pi(1)}, \ldots,{ }^{v \sigma_{n}} / x_{\pi(n)}\right\}$ for some $\varphi$; in words, it is able to mix up the votes before publishing them. However, we don't require this property in general. Protocols that do not respect this property most likely do not respect privacy, but we do not wish to exclude them. 


\subsection{Privacy and receipt-freeness}

Similarly to privacy, receipt-freeness may be formalised as an observational equivalence. In [14], we modeled an election scheme as an applied pi calculus process $V P$, and formalised privacy as the observational equivalence between $V P$ and another version of $V P$ in which two voters $V_{A}$ and $V_{B}$ have swapped their votes. In the remainder of the paper we denote by $V_{A}$ and $V_{B}$ two particular processes such that $V_{A}=V \sigma_{A \mid \operatorname{dom}\left(\sigma_{A}\right) \backslash\{v\}}$ and $V_{B}=$ $V \sigma_{B \mid \operatorname{dom}\left(\sigma_{B}\right) \backslash\{v\}}$ where $\operatorname{dom}\left(\sigma_{A}\right)=\operatorname{dom}\left(\sigma_{B}\right)=f v(V)$. Hence $v$ is the only free variable occuring in $V_{A}$, respectively $V_{B}$. Formally, privacy is defned as follows.

Defnition 4 (Privacy) $A$ voting protocol respects privacy if $S\left[V_{A}\{a / v\} \mid V_{B}\{b / v\}\right] \approx_{\ell} S\left[V_{A}\{b / v\} \mid V_{B}\{a / v\}\right]$.

The intuition is that if an intruder cannot detect if arbitrary honest voters $V_{A}$ and $V_{B}$ swap their votes, then in general he cannot know anything about how $V_{A}$ (or $V_{B}$ ) voted. Note that this defnition is robust even in situations where the result of the election is such that the votes of $V_{A}$ and $V_{B}$ are necessarily revealed: for example, if the vote is unanimous, or if all other voters reveal how they voted and thus allow the votes of $V_{A}$ and $V_{B}$ to be deduced.

We also formalize receipt-freeness using observational equivalence. However, we need to model the fact that $V_{A}$ is willing to provide secret information, i.e., the receipt, to the coercer. We assume that the coercer is in fact the intruder who, as usual in the Dolev-Yao model, controls the public channels. To model $V_{A}$ 's communication with the coercer, we consider that $V_{A}$ executes a voting process which has been modifed: any input and any freshly generated names are forwarded to the coercer.

De£nition 5 (Process $P^{c h}$ ) Let $P$ be a plain process and ch a channel name. We de£ne $P^{c h}$ as follows:

- $0^{\text {ch }} \widehat{=} 0$,

- $(P \mid Q)^{c h} \widehat{=} P^{c h} \mid Q^{c h}$,

- $(\nu n . P)^{c h} \widehat{=} \nu n . \operatorname{out}(c h, n) \cdot P^{c h}$,

- $(\operatorname{in}(u, x) \cdot P)^{c h} \widehat{=} \operatorname{in}(u, x) \cdot \operatorname{out}(c h, x) \cdot P^{c h}$,

- $(\operatorname{out}(u, M) \cdot P)^{c h} \widehat{=} \operatorname{out}(u, M) \cdot P^{c h}$,

- $(! P)^{c h} \widehat{=} ! P^{c h}$,

- $(\text { if } M=N \text { then } P \text { else } Q)^{\text {ch }} \widehat{=}$

$$
\text { if } M=N \text { then } P^{\text {ch }} \text { else } Q^{c h} \text {. }
$$

In the remainder, we assume that $c h \notin f n(P) \cup b n(P)$ before applying the transformation.

Given an extended process $A$ and a channel name $c h$,we need to defne the extended process $A$ \out $(c h, \cdot)$. Intuitively, such a process is as the process $A$, but hiding the ouputs on the channel $c h$.
Defnition 6 (Process $A^{\backslash \text { lout }(\text { ch, }, \cdot)}$ Let $A$ be an extended process. We de£ne $A^{\backslash \text { out }(c h, \cdot)} \widehat{=} \nu$ ch. $(A \mid \operatorname{lin}(c h, x))$.

We are now ready to defne receipt-freeness. Intuitively, a protocol is receipt-free if, for all voters $V_{A}$, the process in which $V_{A}$ votes according to the intruder's wishes is indistinguishable from the one in which she votes something else. As in the case of privacy, we express this as an observational equivalence to a process in which $V_{A}$ swaps her vote with $V_{B}$, in order to avoid the case in which the intruder can distinguish the situations merely by counting the votes at the end. Suppose the coercer's desired vote is $c$. Then we defne receipt-freeness as follows.

Defnition 7 (Receipt-freeness) $A$ voting protocol is receipt-free if there exists a closed plain process $V^{\prime}$, satisfying the two conditions below:

- $V^{\wedge} \backslash$ out (chc, $)$ ) $\approx_{\ell} V_{A}\{a / v\}$, and

- $S\left[V_{A}\left\{{ }^{c} / v\right\}^{c h c} \mid V_{B}\{a / v\}\right] \approx_{\ell} S\left[V^{\prime} \mid V_{B}\{c / v\}\right]$.

$V^{\prime}$ is a process in which voter $V_{A}$ votes $a$ but communicates with the coercer $C$ in order to feign cooperation with him. Thus, the equivalence says that the coercer cannot tell the difference between a situation in which $V_{A}$ genuinely cooperates with him in order to cast the vote $c$ and one in which she pretends to cooperate but actually casts the vote $a$, provided there is some counterbalancing voter that votes the other way around. According to intuition, we have the following proposition.

Proposition 1 Let VP be a voting protocol. $V P$ is receipt-free $\Longrightarrow V P$ respects privacy.

Before we prove this proposition we introduce two lemmas.

Lemma 2 Let $A$ be an extended process, $C$ an evaluation context and ch a channel name. If ch $\notin f n(C[0])$ we have $C[A]^{\backslash \text { out }(\text { ch, },)} \equiv C\left[A^{\backslash \text { out }(c h, \cdot)}\right]$.

Lemma 3 Let $P$ be a closed plain process and ch a channel name such that ch $\notin f n(P) \cup b n(P)$. We have

$$
\left(P^{c h}\right)^{\backslash \text { out }(\operatorname{ch}, \cdot)} \approx_{\ell} P \text {. }
$$

Proof of Proposition 1. By hypothesis, there exists a closed plain process $V^{\prime}$, such that $V^{\prime \text { out }(\text { chc, },)} \approx_{\ell} V_{A}\{a / v\}$ and $S\left[V_{A}\{c / v\}^{c h c} \mid V_{B}\{a / v\}\right] \approx_{\ell} S\left[V^{\prime} \mid V_{B}\{c / v\}\right]$. By applying the evalution context $\nu$ chc. $(|| \operatorname{lin}(\operatorname{chc}, x))$ on both sides and by using Lemmas 2 and 3 , we deduce that: $S\left[V_{A}\left\{{ }^{c} / v\right\} \mid V_{B}\{a / v\}\right] \approx_{\ell} S\left[V^{\prime \backslash \text { out }(\text { chc }, \cdot)} \mid V_{B}\left\{{ }^{c} / v\right\}\right]$. Since $V^{\prime \backslash \text { out }(\text { chc }, \cdot)} \approx_{\ell} V_{A}\{a / v\}$, we easily conclude.

\subsection{Formalising coercion-resistance}

Coercion-resistance is a stronger property as we give the coercer the ability to communicate interactively with the 
voter and not only receive information. In this model, the coercer can for instance prepare the messages he wants the voter to send. As for receipt-freeness, we are going to modify the voter process. However, we give the coercer the possibility to provide the messages the voter should send.

De£nition 8 (Process $P^{c_{1}, c_{2}}$ ) Let $P$ be a plain process and $c_{1}, c_{2}$ be channel names. We de£ne $P^{c_{1}, c_{2}}$ as follows:

- $0^{c_{1}, c_{2}} \widehat{=} 0$,

- $(P \mid Q)^{c_{1}, c_{2}} \widehat{=} P^{c_{1}, c_{2}} \mid Q^{c_{1}, c_{2}}$,

- $(\nu n \cdot P)^{c_{1}, c_{2}} \widehat{=} \nu n \cdot \operatorname{out}\left(c_{1}, n\right) \cdot P^{c_{1}, c_{2}}$,

- $(\operatorname{in}(u, x) \cdot P)^{c_{1}, c_{2}} \widehat{=} \operatorname{in}(u, x) \cdot \operatorname{out}\left(c_{1}, x\right) \cdot P^{c_{1}, c_{2}}$,

- $(\operatorname{out}(u, M) \cdot P)^{c_{1}, c_{2}} \widehat{=} \operatorname{in}\left(c_{2}, x\right) \cdot \operatorname{out}(u, x) \cdot P^{c_{1}, c_{2}}$ where $x$ is a fresh variable,

- $(! P)^{c_{1}, c_{2}} \widehat{=} ! P^{c_{1}, c_{2}}$,

- (if $M=N$ then P else $Q)^{c_{1}, c_{2}} \widehat{=}$

$$
\text { if } M=N \text { then } P^{c_{1}, c_{2}} \text { else } Q^{c_{1}, c_{2}} \text {. }
$$

As a £rst approximation, we could try to de£ne coercionresistance in the following way:

$$
S\left[V_{A}\{c / v\}^{c_{1}, c_{2}} \mid V_{B}\{a / v\}\right] \approx_{\ell} S\left[V^{\prime} \mid V_{B}\{c / v\}\right] .
$$

This defnition has an obvious problem as the coercer could oblige $V_{A}\{c / v\}^{c_{1}, c_{2}}$ to vote $c^{\prime} \neq c$. In that case, the process $V_{B}\left\{{ }^{c} / v\right\}$ would not counterbalance the outcome to avoid a trivial way of distinguishing.

To properly de£ne coercion-resistance, we de£ne a new simulation relation which allows the second voting process on the right-hand side to dynamically adapt its vote to correspond to the coercer's choice. Before de£ning this relation itself we have to introduce some more notation.

Defnition $9\left(X_{A}\right)$ Given an extended process $A$, we de£ne $X_{A}$ to be the set of unbound variables that are not de£ned by an active substitution, i.e., $X_{A}=f v(A) \backslash \operatorname{dom}(\phi(A))$.

Example 3 Consider the following extended process B:

$$
\begin{gathered}
\operatorname{out}\left(c_{1}, x_{1}\right) \cdot \operatorname{out}\left(c_{2}, x_{2}\right)\left|\operatorname{in}\left(c_{1}, y_{1}\right) \cdot \operatorname{in}\left(c_{2}, y_{2}\right)\right| \\
\left\{\operatorname{enc}\left(z_{1}, z_{2}\right) / x_{1}\right\} \mid\left\{\operatorname{enc}\left(z_{1}, z_{3}\right) / x_{2}\right\} .
\end{gathered}
$$

We have $X_{B}=\left\{z_{1}, z_{2}, z_{3}\right\}$.

De£nition $10\left(X_{A}\left(A \sigma \stackrel{(\alpha)}{\rightarrow} A^{\prime}\right)\right)$ Let $A$ be an extended process and $\sigma$ be a substitution closing $A$, i.e., $\operatorname{dom}(\sigma)=X_{A}$. We de£ne $X_{A}\left(A \sigma \rightarrow A^{\prime}\right)$ (resp. $X_{A}\left(A \sigma \stackrel{\alpha}{\rightarrow} A^{\prime}\right)$ ) to be the variables in $X_{A}$ that are bound by $\sigma$ during the internal reduction $A \sigma \rightarrow A^{\prime}$ (resp. labeled transition $A \sigma \stackrel{\alpha}{\rightarrow} A^{\prime}$ ). More formally, see Figure 1.
This defnition can be extended naturally to any sequences of reduction steps.

Example 4 Let $B$ be the process de£ned in Example 3. We have $\phi(B)=\left\{\operatorname{enc}\left(z_{1}, z_{2}\right) / x_{1}\right.$,enc $\left.\left(z_{1}, z_{3}\right) / x_{2}\right\}$. Let $\sigma=\left\{z_{1} \mapsto a, z_{2} \mapsto b, z_{3} \mapsto c\right\}$. Let $B_{1}=\operatorname{out}\left(c_{2}, x_{2}\right)$ $\operatorname{in}\left(c_{2}, y_{2}\right)\left|\left\{\operatorname{enc}(a, b) / x_{1}\right\}\right|\left\{\operatorname{enc}\left(a, z_{3}\right) / x_{2}\right\}$ and $B_{2}=$ $\left\{\right.$ enc $\left.(a, b) / x_{1}\right\} \mid\left\{\right.$ enc $\left.(a, c) / x_{2}\right\}$. We have $X_{B}(B \sigma \rightarrow$ $\left.B_{1} \sigma\right)=\left\{z_{1}, z_{2}\right\}$ and $X_{B_{1}}\left(B_{1} \sigma \rightarrow B_{2}\right)=\left\{z_{3}\right\}$.

De£nition 11 (Adaptive simulation $\left(\preceq_{a}\right)$ ) Adaptive simulation is the largest relation $\mathcal{R}$ on extended processes $A$ and $B$, such that $A \mathcal{R} B$ implies that for all $\sigma_{A}$ such that $\operatorname{dom}\left(\sigma_{A}\right)=X_{A}$

1. $A \sigma_{A} \approx_{s} B \sigma_{B}$ for some substitution $\sigma_{B}$ with $\operatorname{dom}\left(\sigma_{B}\right)=X_{B}$;

2. if $A \sigma_{A} \rightarrow A^{\prime} \sigma_{A}$ then there exists $\sigma_{B}$ with $\operatorname{dom}\left(\sigma_{B}\right)=X_{B}$ such that

(a) there exists $B^{\prime}$ such that $B \sigma_{B} \rightarrow^{*} B^{\prime} \sigma_{B}$ and $A^{\prime} \theta_{A} \mathcal{R} B^{\prime} \theta_{B}$ where $\theta_{A}=\left.\sigma_{A}\right|_{X_{A}}\left(A \sigma_{A} \rightarrow A^{\prime} \sigma_{A}\right)$ and $\theta_{B}=\left.\sigma_{B}\right|_{X_{B}\left(B \sigma_{B} \rightarrow * B^{\prime} \sigma_{B}\right)}$;

(b) if $B \sigma_{B} \rightarrow B^{\prime} \sigma_{B}$ then there exists $A^{\prime}$ such that $A \sigma_{A} \rightarrow^{*} A^{\prime} \sigma_{A}$ and $A^{\prime} \theta_{A} \mathcal{R} B^{\prime} \theta_{B}$ where

$\theta_{A}=\left.\sigma_{A}\right|_{X_{A}\left(A \sigma_{A} \rightarrow^{*} A^{\prime} \sigma_{A}\right)}$ and $\theta_{B}=\left.\sigma_{B}\right|_{X_{B}\left(B \sigma_{B} \rightarrow B^{\prime} \sigma_{B}\right)}$.

3. if $A \sigma_{A} \stackrel{\alpha}{\rightarrow} A^{\prime} \sigma_{A}$ then there exists $\sigma_{B}$ with $\operatorname{dom}\left(\sigma_{B}\right)=X_{B}$ such that

(a) there exists $B^{\prime}$ such that $B \sigma_{B} \rightarrow \stackrel{*}{\rightarrow} \rightarrow{ }^{*} B^{\prime} \sigma_{B}$ and $A^{\prime} \theta_{A} \mathcal{R} B^{\prime} \theta_{B}$ where $\theta_{A}=\left.\sigma_{A}\right|_{X_{A}\left(A \sigma_{A} \stackrel{\alpha}{\rightarrow} A^{\prime} \sigma_{A}\right)}$ and
$\theta_{B}=\left.\sigma_{B}\right|_{X_{B}\left(B \sigma_{B} \rightarrow * \stackrel{\alpha}{\rightarrow} \rightarrow * B^{\prime} \sigma_{B}\right)} ;$

(b) if $B \sigma_{B} \stackrel{\alpha}{\rightarrow} B^{\prime} \sigma_{B}$ then there exists $A^{\prime}$ such that $A \sigma_{A} \rightarrow^{*} \stackrel{\alpha}{\longrightarrow} \longrightarrow^{*} A^{\prime} \sigma_{A}$ and $A^{\prime} \theta_{A} \mathcal{R} B^{\prime} \theta_{B}$ where $\theta_{A}=\left.\sigma_{A}\right|_{X_{A}\left(A \sigma_{A} \rightarrow * \stackrel{\alpha}{\rightarrow} \rightarrow * A^{\prime} \sigma_{A}\right)}$ and $\theta_{B}=\left.\sigma_{B}\right|_{X_{B}\left(B \sigma_{B} \stackrel{\alpha}{\rightarrow} B^{\prime} \sigma_{B}\right)}$.

Intuitively, adaptive simulation models the fact that no matter how the process $A$ is closed and no matter how the environment reacts, $B$ can be closed, such that the processes are indistinguishable. The existential quantifcation on $\sigma_{B}$ follows the reductions of $A$, in order to let $B$ adapt to the inputs of the environment. While the $(a)$ parts ensure that $B$ indeed simulates $A$, the $(b)$ parts ensure that $B$ cannot "do anything more" than $A$. Finally, by the means of $\theta_{A}$ and $\theta_{B}$ we progressively close $A$ and $B$, so that once their reductions depend on some variables, the value of these variables cannot be changed any more, i.e., they have to stick to their choices and cannot undo them anymore. Note that if $A$ and $B$ are closed processes $A \approx_{\ell} B$ if and only if $A \preceq_{a} B$. Moreover adaptive simulation is transitive and enjoys several other useful properties stated below. 


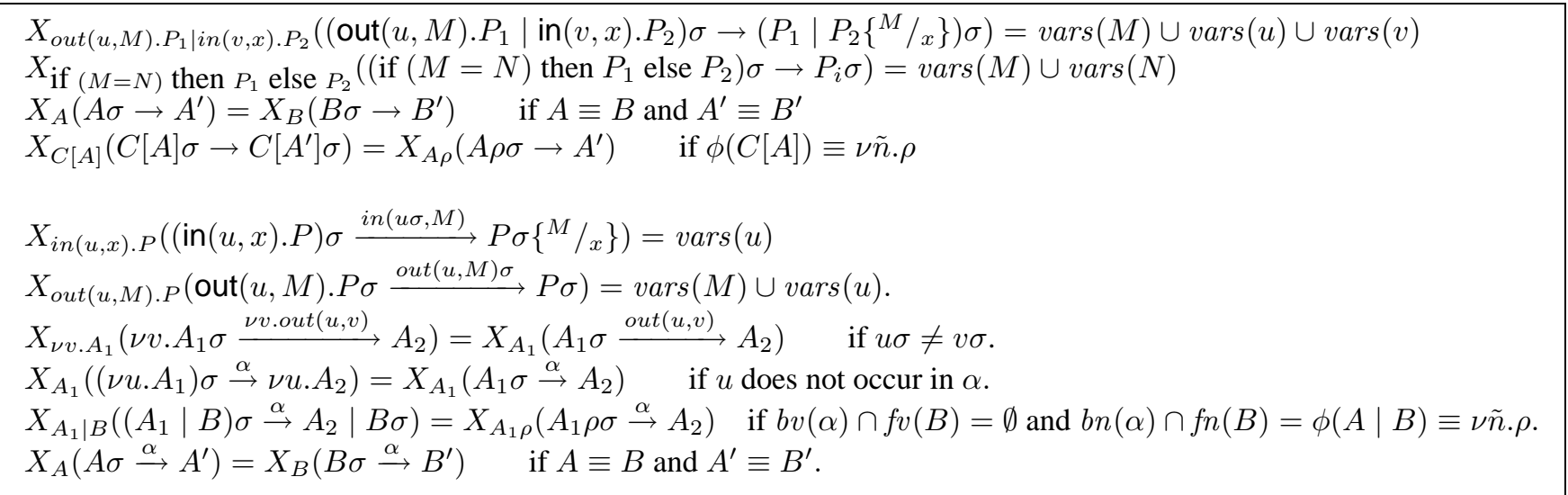

Figure 1: $X_{A}\left(A \sigma \stackrel{(\alpha)}{\rightarrow} A^{\prime}\right)$

Lemma 4 Let $A$ and $B$ be two extended processes. If for all $\rho_{A}$ with $\operatorname{dom}\left(\rho_{A}\right)=X_{A}$ there exists $\rho_{B}$ with $\operatorname{dom}\left(\rho_{B}\right)=X_{B}$ such that $A \rho_{A} \approx_{\ell} B \rho_{B}$, then $A \preceq_{a} B$.

Note that the converse is not true. Consider the two processes $A$ and $B$ described below:

$A=\nu c .\left(\operatorname{in}\left(c_{1}, x\right) \cdot \operatorname{out}(c, a) \cdot \operatorname{out}\left(c_{2}, x\right) \mid \operatorname{in}(c, z) \cdot \operatorname{out}\left(c_{2}, a\right)\right)$; $B=\nu c$. $\left(\operatorname{in}\left(c_{1}, x\right) \cdot \operatorname{out}(c, a) \cdot \operatorname{out}\left(c_{2}, a\right) \mid \operatorname{in}(c, z) \cdot \operatorname{out}\left(c_{2}, y\right)\right)$. We have that $A \preceq_{a} B$. However there is no $\rho_{B}$ such that for all $\rho_{A}$ we have $A \rho_{A} \approx_{\ell} B \rho_{B}$.

Corollary 1 Let $A$ and $B$ be two extended processes such that $X_{A}=X_{B}$. If for all substitution $\rho$ with $\operatorname{dom}(\rho)=$ $X_{A}=X_{B}$ we have $A \rho \approx_{\ell} B \rho$, then $A \preceq_{a} B$.

We say that an evaluation context is strict, if the hole does not appear under a restriction of a variable.

Lemma 5 Let $A, B$ be two extended processes and $C$ a strict evaluation context, such that $f v(C[0])=\emptyset$. We have

$$
A \preceq_{a} B \Longrightarrow C[A] \preceq_{a} C[B] .
$$

Note that in Lemma 5 we cannot consider any open context where $f v(C[0]) \neq \emptyset$. Consider for example $A=\left\{{ }^{a} / x_{1}\right\}$ and $B=\left\{{ }^{y} / x_{1}\right\}$. Then we have $A \preceq_{a} B$, while $A \mid$ $\left\{y / x_{2}\right\} \npreceq_{a} B \mid\left\{y / x_{2}\right\}$. One might want to relax this condition to $f v(C[0]) \cap f v(B)=\emptyset$. However, this stronger version is not needed in the remainder and would complicate the proofs. For a similar reason we also restrict ourselves to strict evaluation contexts, as $\nu y . A \npreceq a \nu y . B$.

We are now ready to defne coercion-resistance.

Defnition 12 (Coercion-resistance) A voting protocol is coercion-resistant if there exists a closed extended process $V^{\prime}$ and a strict evaluation context $C$ such that

- $S\left[V_{A}\{c / v\}^{c_{1}, c_{2}} \mid V_{B}\{a / v\}\right] \preceq_{a} S\left[V^{\prime} \mid V_{B}\{x / v\}\right]$,

- $\nu c_{1}, c_{2} . C\left[V_{A}\{c / v\}^{c_{1}, c_{2}}\right] \approx_{\ell} V_{A}\{c / v\}^{c h c}$,
- $\nu c_{1}, c_{2} \cdot C\left[V^{\prime}\right]^{\backslash o u t}(\operatorname{chc}, \cdot) \approx_{\ell} V_{A}\{a / v\}$,

where $x$ is a fresh free variable.

The intuition of this defnition is that whenever the coercer requests a given vote on the left-hand side, then $V_{B}$ can adapt his vote on the right-hand side and counterbalance the outcome. However, we need to avoid the case where $V^{\prime}=V_{A}\left\{{ }^{c} / v\right\}^{c_{1}, c_{2}}$ letting $V_{B}$ vote $a$. Therefore we require that when we apply a context $C$, intuitively the coercer, requesting $V_{A}\left\{{ }^{c} / v\right\}^{c_{1}, c_{2}}$ to vote $c, V^{\prime}$ in the same context votes $a$. There may be circumstances where $V^{\prime}$ may need not to cast a vote that is not $a$. We discuss those in Section 3.4.

Proposition 2 Let VP be a voting protocol. $V P$ is coercion-resistant $\Longrightarrow V P$ is receipt-free.

Proof. We need to show that there exists $V^{\prime \prime}$ satisfying the conditions of receipt-freeness. Let $V^{\prime \prime}=\nu c_{1}, c_{2} . C\left[V^{\prime}\right]$. we have to show that

$$
S\left[V_{A}\left\{{ }^{c} / v\right\}^{c h c} \mid V_{B}\{a / v\}\right] \approx_{\ell} S\left[V^{\prime \prime} \mid V_{B}\{c / v\}\right] .
$$

Thanks to Lemma 5, we know that:

$$
\begin{gathered}
\nu c_{1}, c_{2} \cdot C\left[S\left[V_{A}\left\{{ }^{c} / v\right\}^{c_{1}, c_{2}} \mid V_{B}\left\{{ }^{a} / v\right\}\right]\right] \\
\preceq_{a} \\
\nu c_{1}, c_{2} \cdot C\left[S\left[V^{\prime} \mid V_{B}\left\{{ }^{x} / v\right\}\right]\right] .
\end{gathered}
$$

Moreover, we have

$$
\begin{aligned}
& \nu c_{1}, c_{2} \cdot C\left[S\left[V_{A}\{c / v\}^{c_{1}, c_{2}} \mid V_{B}\{a / v\}\right]\right] \\
\equiv & S\left[\nu c_{1}, c_{2} \cdot C\left[V_{A}\{c / v\}^{c_{1}, c_{2}}\right] \mid V_{B}\{a / v\}\right] \text { by Lemma } 1 \\
\approx_{\ell} & S\left[V_{A}\{c / v\}^{c h c} \mid V_{B}\{a / v\}\right] \text { by hypothesis }
\end{aligned}
$$

Hence, we deduce that $S\left[V_{A}\left\{{ }^{c} / v\right\}^{c h c} \mid V_{B}\{a / v\}\right] \preceq_{a} \nu c_{1}, c_{2} . C\left[S\left[V^{\prime} \mid V_{B}\{x / v\}\right]\right]$.

For any substitution $\rho$ such that $\operatorname{dom}(\rho)=\{x\}$ we have $\nu c_{1}, c_{2} . C\left[S\left[V^{\prime} \mid V_{B}\left\{{ }^{x \rho} / v\right\}\right]\right] \approx_{\ell} S\left[V^{\prime \prime} \mid V_{B}\left\{{ }^{x \rho} / v\right\}\right]$.

Using Corollary 1 we obtain that $\nu c_{1}, c_{2} . C\left[S\left[V^{\prime} \mid V_{B}\left\{{ }^{x} / v\right\}\right]\right] \preceq_{a} S\left[V^{\prime \prime} \mid V_{B}\left\{{ }^{x} / v\right\}\right]$. 
Thanks to the transitivity of $\preceq_{a}$, we deduce that

$$
S\left[V_{A}\left\{{ }^{c} / v\right\}^{c h c} \mid V_{B}\{a / v\}\right] \preceq_{a} S\left[V^{\prime \prime} \mid V_{B}\{x / v\}\right] .
$$

By the defnition of a voting process, we have

$$
S\left[V_{A}\{c / v\}^{c h c} \mid V_{B}\{a / v\}\right]\left(\rightarrow^{*} \stackrel{\alpha}{\rightarrow}\right)^{*} S^{\prime}
$$

where $\phi\left(S^{\prime}\right) \equiv \varphi^{\prime} \mid\left\{a / x_{1}, c / x_{2}\right\}$ for some $\varphi^{\prime}$. Therefore there exists a substitution $\sigma$ with $x \in \operatorname{dom}(\sigma)$ such that

$$
S\left[V^{\prime \prime} \mid V_{B}\{x / v\}\right] \sigma\left(\rightarrow^{*} \stackrel{\alpha}{\rightarrow}\right)^{*} S^{\prime \prime}
$$

where $\phi\left(S^{\prime \prime}\right) \equiv \varphi^{\prime \prime} \mid\left\{{ }^{a} / x_{1},{ }^{c} / x_{2}\right\}$ for some $\varphi^{\prime \prime}$. Since $V^{\prime \prime \backslash \text { out }(\text { chc,. })} \approx_{\ell} V_{A}\{a / v\}, V$ does not output $c$ on a channel it shares with $S[-]$. From equation (1) and the fact that $c$ does not occur in $S$ []], it must be that $\sigma(x)=c$ in all evolutions of $S\left[V^{\prime \prime} \mid V_{B}\left\{{ }^{x} / v\right\}\right] \sigma$ preserving the adaptive simulation. Hence the result.

\subsection{Fault attacks}

Looking closely at the defnition of coercion-resistance, one may notice that the intruder could have an unexpected strategy to distinguish the left-hand process from the righthand process. In protocols in which the voter is expected to submit certain messages to the election administrators, the arrangement between the voter and the coercer may involve the coercer preparing those messages for the voter. In this case, the coercer may submit messages to $V_{A}\left\{{ }^{c} / v\right\}^{c_{1}, c_{2}}$ with the pure intention to block the process on the left-hand side of the relation. If $V^{\prime}$ is unable to detect that the message is incoherent, the process will not block on the right-hand side, thus yielding an observable difference. Our defnition therefore allows $V^{\prime}$ to block too and not to vote if $V^{\prime}$ thinks that the messages sent by the coercer would block the protocol.

In a real-life scenario the intruder may use this method to test the loyalty of $V_{A}$ in a probabilistic way. For example, in one out of every one hundred coerced voters, the coercer might submit such a garbage message to the voter. If the voter is not genuinely cooperating with him by submitting the garbage message, and instead casts his own vote successfully, the attacker can perceive a difference.

We argue that this attack, if successful, is an attack against coercion-resistance, since it means that the voter knows that the coercer has the ability to detect whether the voter is cooperating with him or not. Launching the attack costs the coercer that particular vote, but it is a means of applying pressure on the voter to cooperate.

Our defnition of coercion-resistance is correct with respect to this kind of attack. That is, a protocol which is vulnerable to the attack is not coercion-resistant according to our defnition. A protocol is coercion-resistant if $V^{\prime}$ can be chosen to mimic $V_{A}\{c / v\}^{c_{1}, c_{2}}$. Thus, if $V^{\prime}$ can detect that the message from the coercer is incoherent, she can act in order to block the protocol, preserving the relation.

\section{Example}

In this section we apply the formalization, described in the previous section to a simplifed version of the Lee et al. protocol [15]. One of the main advantages of this protocol is that it is vote and go: voters need participate in the election only once, in contrast with [10], where all voters have to £nish a £rst phase before any of them can participate in the second phase.

We simplifed the protocol in order to concentrate on the aspects that are important with respect to receipt-freeness and coercion-resistance. In particular we do not consider distributed authorities. Nevertheless, the protocol is neither trivial to model nor to analyse and illustrates well subtle issues in coercion-resistance.

\subsection{Description of the protocol}

The protocol relies on two less usual cryptographic primitives: re-encryption and designated verifer proofs (DVP) of re-encryption. We start by explaining these primitives.

A re-encryption of a ciphertext (obtained using a randomized encryption scheme) changes the random coins, without changing or revealing the plaintext. In the ElGamal scheme for instance, if $(x, y)$ is the ciphertext, this is simply done by computing $\left(x g^{r}, y h^{r}\right)$, where $r$ is a random number, and $g$ and $h$ are the subgroup generator and the public key respectively. Note that neither the creator of the original ciphertext nor the person re-encrypting knows the random coins used in the re-encrypted ciphertext, for they are a function of the coins chosen by both parties. In particular, a voter cannot reveal the coins to a potential coercer who could use this information to verify the value of the vote, by ciphering his expected vote with these coins.

A DVP of the re-encryption proves that the two ciphertexts contain indeed the same plaintext. However, a designated verifer proof only convinces one intended person, e.g., the voter, that the re-encrypted ciphertext contains the original plaintext. In particular this proof cannot be used to convince the coercer. Technically, this is achieved by giving the designated verifer the ability to simulate the transcripts of the proof.

Our simplifed protocol can be described in three steps. Firstly, the voter encrypts his vote with the collector's public key, signs the encrypted vote and sends it to an administrator on a private channel. The administrator checks whether the voter is a legitimate voter and has not voted yet. Then the administrator re-encrypts the given ciphertext, signs it and sends it back to the voter. The administrator also provides a DVP that the two ciphertexts contain indeed the same plaintext. In practice, this £rst stage of the protocol can be done using a voting booth where eligibility of the voter is tested at the entrance of the booth. The booth con- 


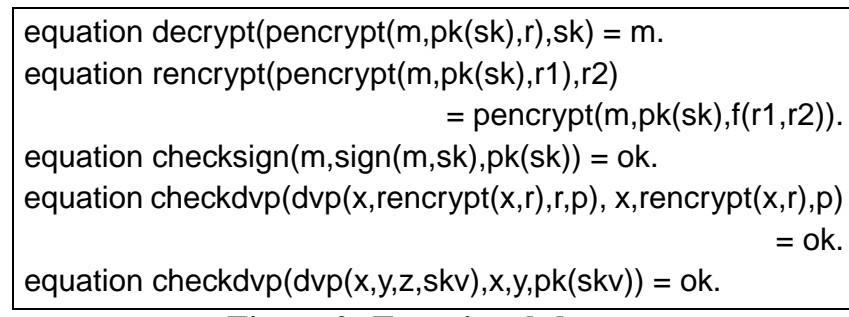

Figure 2: Equational theory

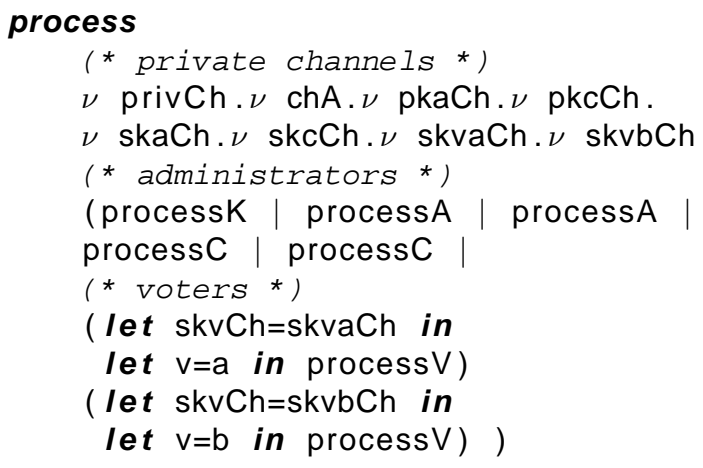

\section{Process 1. Main process}

tains a tamper-proof device which performs re-encryptions, signatures and DVP proofs. Then, the voter sends (via an anonymous channel) the re-encrypted vote, which has been signed by the administrator to the public board. Finally, the collector checks the administrator's signature on each of the votes and, if valid, decrypts the votes and publishes the £nal results.

\subsection{Applied pi calculus model}

Cryptographic primitives as an equational theory. The equational theory is represented in Figure 2. The functions and equations that handle public keys, probabilistic encryption and digital signature are as usual. To model reencryption we add a function rencrypt, that permits us to obtain a different encryption of the same message with another random coin which is function of the original one and the one used during the re-encryption. We also add a pair of functions dvp and checkdvp: dvp permits us to build a designated verifer proof of the fact that a message is a re-encryption of another one and checkdvp allows the designated verifer to check that the proof is valid. Note that checkdvp also succeeds for a fake dvp created using the designated veri£er's private key.

Main (Process 1). The main process sets up private channels and specifes how the processes are combined in parallel. Most of the private channels are for key distribution.

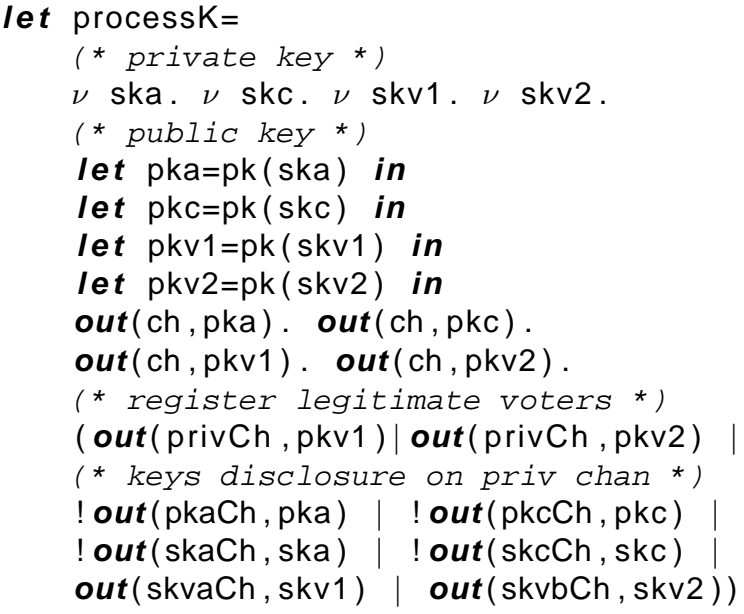

Process 2. Administrator for keying material

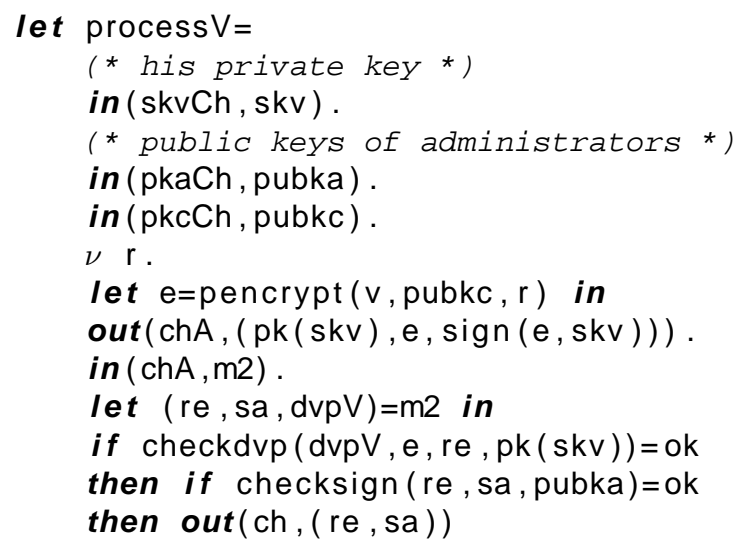

\section{Process 3. Voter process}

The private channel chA is a private channel between the administrator and voters. This is motivated by the fact that the administrator corresponds to a tamper-proof hardware device in this protocol. For ease of presentation, we only model the protocol for two voters and launch two copies of the administrator and collector process, one for each voter.

Keying material (Process 2). Our model includes a dedicated process for generating and distributing keying material modeling a PKI. Additionally, this process registers legitimate voters and also distributes the public keys of the election authorities to legitimate voters: this is modeled using restricted channels so that the attacker cannot provide false public keys.

Voter (Process 3). First, each voter obtains his secret key from the PKI as well as the public keys of the election au- 


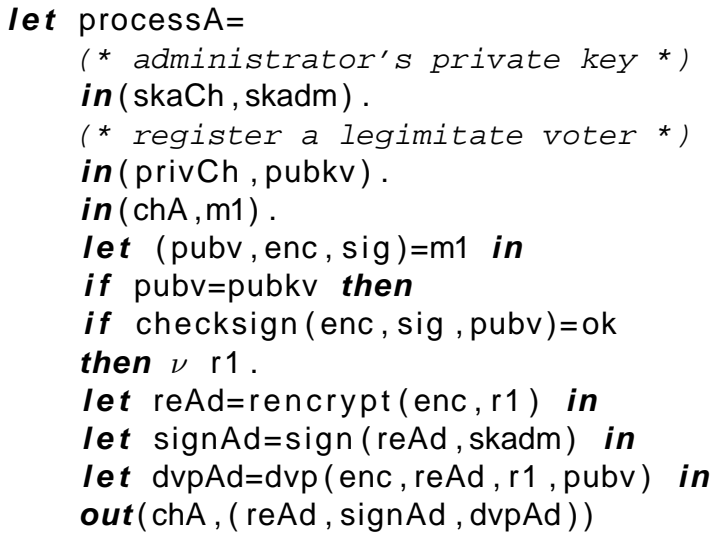

Process 4. Administrator process

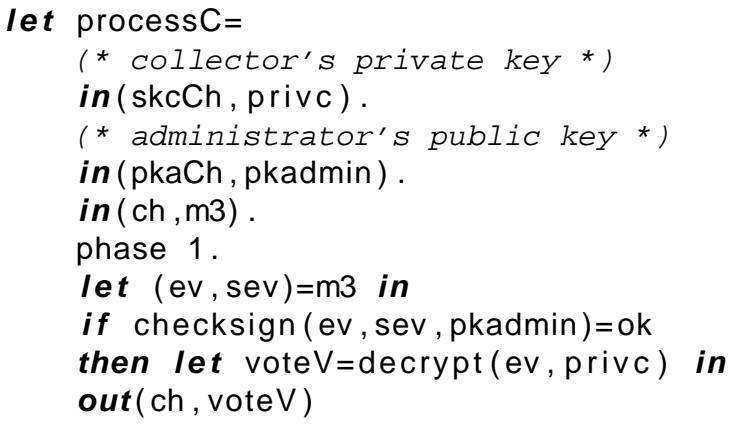

\section{Process 5. Collector process}

thorities. Then, a fresh random number is generated to encrypt his vote with the public key of the collector. Next, he signs the result and sends it on a private channel to the administrator. If the voter has been correctly registered, he obtains from the administrator, a re-encryption of his vote signed by the administrator together with a designated verifer proof of the fact that this re-encryption has been done correctly. If this proof is correct, then the voter sends his reencrypted vote signed by the administrator to the collector.

Administrator (Process 4). The administrator £rst receives through a private channel his own public key as well as the public key of a legitimate voter. The received public key has to match the voter who is trying to get a reencryption of his vote signed by the administrator. The administrator has also to prove to the voter that he has done the re-encryption properly. For this, he builds a designated verifer proof which will be only convincing for the voter.

Collector (Process 5). To model the collector, we use the phase command, introduced in the ProVerif tool [5], as a global synchronisation command, i.e., all processes £nish

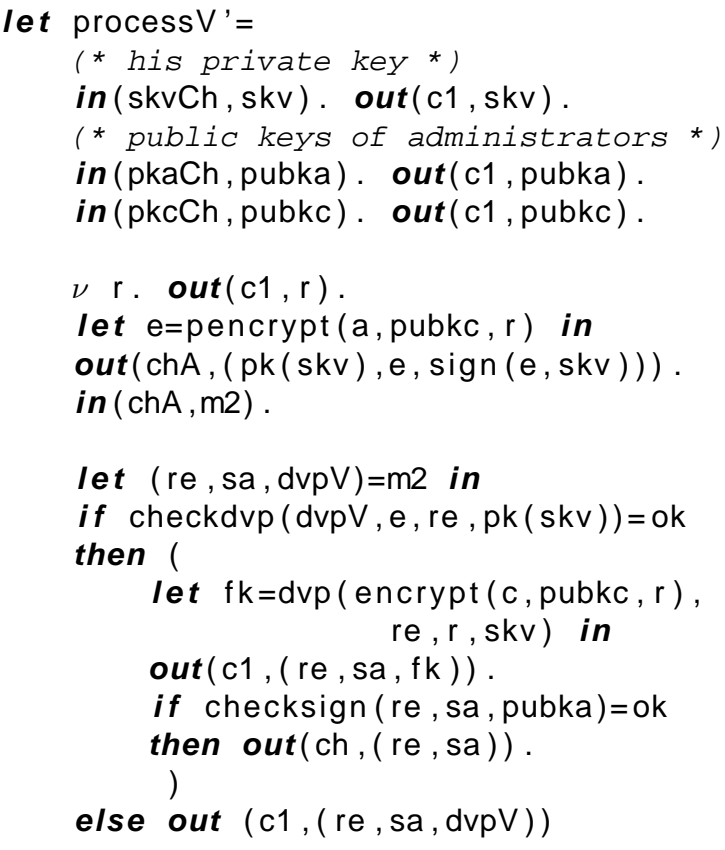

Process 6. Process $V^{\prime}$ - receipt-freeness

the instructions of a given phase before any of them can start the following phase. This separation is crucial for receiptfreeness (and hence coercion-resistance) to hold. First, the collector receives all the signed ballots. He checks the signature and decrypts the result with his private key to obtain the value of the vote in order to publish the results.

\subsection{Receipt-freeness, coercion-resistance}

We do not give full formal proofs here but only the ideas on how to construct the $V^{\prime}$ processes. The aim is merely to illustrate the defnitions. We denote $V_{A}=$ $V\{s k v a C h / s k v C h\}$ and $V_{B}=V\{s k v b C h / s k v C h\}$.

Receipt-freeness. To show receipt-freeness one needs to construct a process $V^{\prime}$ which successfully can fake all secrets to a coercer. The idea is for $V^{\prime}$ to vote $a$, but when outputting secrets to the coercer $V^{\prime}$ prepares all outputs as if he was voting $c$. The crucial part is that, using his private key, he provides a fake dvp stating that the actual reencryption of the encryption of vote $a$ is a re-encryption of the encryption of vote $c$. Given our equational theory, the two resulting frames are statically equivalent as for both the real and the fake dvp, checkdvp would give ok.

Process 6 shows a possible $V^{\prime}$. To prove receiptfreeness, we need to show

- $V^{\prime \text { out }(\operatorname{chc}, \cdot)} \approx_{\ell} V_{A}\{a / v\}$, and

- $S\left[V_{A}\{c / v\}^{c h c} \mid V_{B}\{a / v\}\right] \approx_{\ell} S\left[V^{\prime} \mid V_{B}\{c / v\}\right]$. 
The Erst one may be seen informally by considering $V^{\prime}$ with the "out $(\mathrm{c} 1, \ldots)$ " commands removed, and comparing it visually with $V_{A}$. To see the second labelled bisimulation, one can informally consider all the executions of each side. $S$ consists of the Main process, and therefore includes process K, the two processA's, and the two processC's, but it has a hole for the two voter processes. As shown above, the hole is flled by $V_{A}\{c / v\}^{c h c} \mid V_{B}\{a / v\}$ on the left and by $V^{\prime} \mid V_{B}\left\{{ }^{c} / v\right\}$ on the right. Executions of $V_{A}\left\{{ }^{c} / v\right\}^{c h c}$ are matched with those of $V^{\prime}$; similarly, $V_{B}\{a / v\}$ on the left is matched with $V_{B}\left\{{ }^{c} / v\right\}$ on the right. The attacker could try to make one side block, but this will make the other one block too. Both left and right sides result in votes for $a$ and $c$; thus, when fully executed, if one side results in a process whose frame contains the active substitutions $\left\{a / x_{1}, c / x_{2}\right\}$ then the other one can be executed that way too.

Coercion-resistance. The construction of $V^{\prime}$ is similar to the one for receipt-freeness. However, for coercionresistance the coercer also provides the inputs for the messages to send out. If the coercer prepares messages corresponding to a given vote, we fake the outputs as previously and know that the non-coerced voter will counterbalance the outcome, by adaptively choosing the same vote. The process $V^{\prime}$ and the strict evaluation context $C$ required for the defnition of coercion resistance are shown in Processes 7 and 8. Similar reasoning to that used above (for receipt freeness) can be used here, to establish whether the three conditions

- $\nu c_{1}, c_{2} . C\left[V_{A}\left\{{ }^{c} / v\right\}^{c_{1}, c_{2}}\right] \approx_{\ell} V_{A}\left\{{ }^{c} / v\right\}^{c h c}$,

- $\nu c_{1}, c_{2} . C\left[V^{\prime}\right]^{\backslash \text { out }(c h c, \cdot)} \approx_{\ell} V_{A}\left\{{ }^{a} / v\right\}$,

$$
\text { - } S\left[V_{A}\left\{{ }^{c} / v\right\}^{c_{1}, c_{2}} \mid V_{B}\left\{{ }^{a} / v\right\}\right] \preceq_{a} S\left[V^{\prime} \mid V_{B}\left\{^{x} / v\right\}\right] \text {, }
$$

hold for coercion resistance. The frst two are straightforward, but reasoning about the third one reveals some subtleties about details of how the protocol is implemented.

The attacker can observe a difference between both sides if he schedules the processes so that $V_{A}\{c / v\}^{c_{1}, c_{2}}$ is delayed on the left while $V_{B}\{a / v\}$ is allowed to proceed. If this happens, we cannot £nd the substitution for $x$ required for the adaptive simulation so that $V_{B}\{x / v\}$ can proceed. The attacker will observe a difference because $V_{B}\{a / v\}$ will send his vote on the left, while $V_{B}\left\{{ }^{x} / v\right\}$ will not. To prevent this attack, we can make the voters report their votes along a private channel instead of a public one (last line of Process 3). This means that the protocol could not be used over the internet if one wants to guarantee coercion resistance.

Another way to attack coercion resistance is to use the fault attacks described in section 3.4. Here, the coercer provides a badly formatted input. The voter should detect this

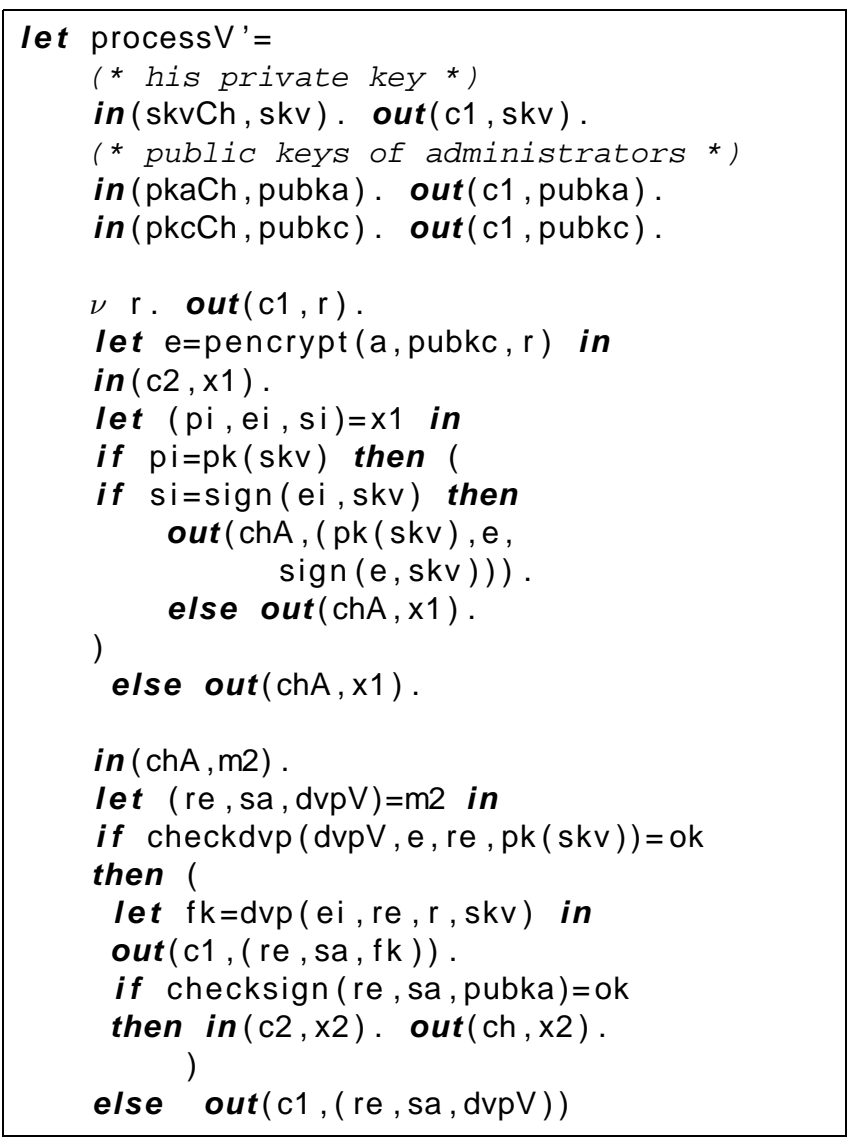

\section{Process 7. Process $V^{\prime}$ - coercion-resistance}

and just follow the instructions to avoid the fault attack. Invalid signatures are easy to detect. However, the case where the frst encrypted vote sent to the administrator is an invalid encryption is more diffcult to handle as $V^{\prime}$ cannot detect it. Here we can consider several cases depending on the details of the implementation, namely whether decryption is possible on every bitstring. If so (as in our equational theory), then the other voter could counterbalance by choosing $x$ to be the decryption of the given garbage message. At the tallying stage this would indeed result in an invalid vote on both sides. If one considers encryption with integrity checking, which one could model in applied pi calculus by adding an explicit checking equation, then this protocol would not be coercion-resistant. This is because the non-coerced is only allowed to choose the value of its vote, but in other respects it follows the protocol and in particular encrypts the chosen vote correctly. Therefore it cannot mimick the coerced voter who sends an invalid vote. Thus the collector blocks when trying to decrypt the vote for the coerced voter, but not for $V_{B}$, resulting in an observable difference. 


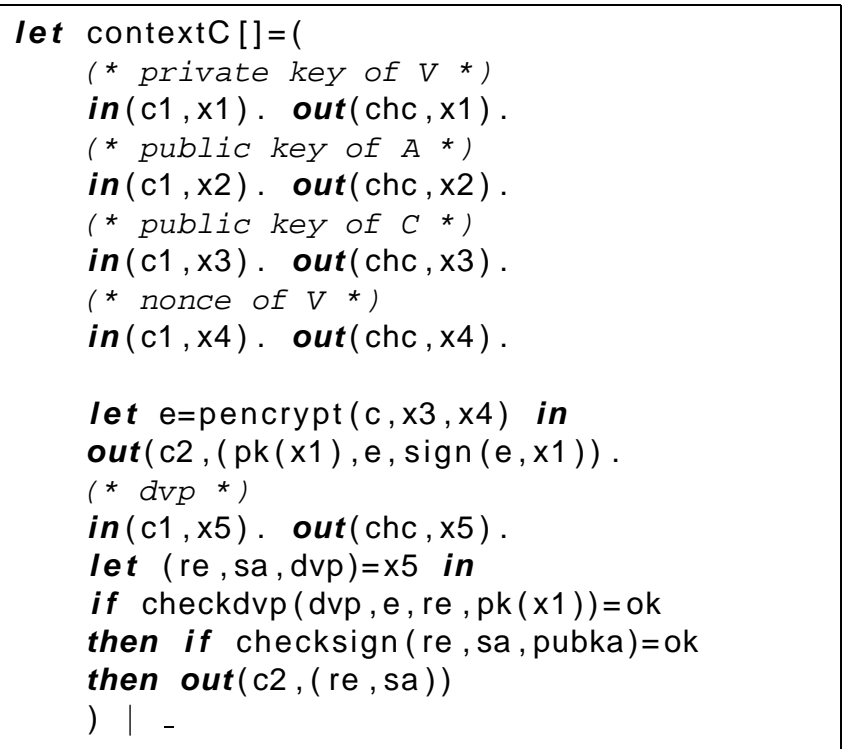

\section{Process 8. Context $C$ - coercion-resistance}

\section{Conclusion}

In this paper we studied two particular anonymity properties of election protocols: receipt-freeness and coercionresistance. Although the properties are modelled using these different relations, we can prove that, according to the intuition, coercion-resistance implies receipt-freeness which itself implies privacy. Finally we illustrate the definitions on a simplifed version of the Lee et al. protocol.

As future work we would like to automate the verifcation of observational equivalence. Although the ProVerif tool can in many special cases prove observational equivalence, it is not able to do so for privacy or the more elaborated properties of this paper. We foresee to investigate automatic verifcation of observational equivalence at least for a £nite number of sessions (not authorizing replication) and for restricted classes of equational theories.

\section{References}

[1] M. Abadi, B. Blanchet, and C. Fournet. Just fast keying in the pi calculus. In Proc. 13th European Symposium On Programming (ESOP'04), volume 2986 of LNCS, pages 340 354, Barcelona, Spain, 2004. Springer.

[2] M. Abadi and C. Fournet. Mobile values, new names, and secure communication. In Proc. 28th ACM Symposium on Principles of Programming Languages (POPL'01), pages 104-115, London, UK, 2001. ACM.

[3] J. Benaloh. Verifable Secret Ballot Elections. PhD thesis, Yale University, 1987.

[4] J. Benaloh and D. Tuinstra. Receipt-free secret-ballot elections (extended abstract). In Proc. 26th Annual Sympo- sium on Theory of Computing (STOC'94), pages 544-553, Montréal, Québec, 1994. ACM Press.

[5] B. Blanchet. ProVerif: Automatic Cryptographic Protocol Verifer User Manual, 2005.

[6] D. Chaum. Elections with unconditionally-secret ballots and disruption equivalent to breaking RSA. In Proc. of Advances in Cryptology (Eurocrypt'88), volume 330 of LNCS, pages 177-182, Davos, Switzerland, 1988. Springer.

[7] D. Chaum, P. Y. A. Ryan, and S. Schneider. A practical, voter-verifable election scheme. In Proc. 10th European Symposium On Research In Computer Security (ESORICS'05), volume 3679 of $L N C S$, pages 118-139, Milan, Italy, 2005. Springer.

[8] S. Delaune, S. Kremer, and M. D. Ryan. Coercion-resistance and receipt-freeness in electronic voting. Research Report LSV-06-08, Laboratoire Spécifcation et Vérifcation, ENS Cachan, France, Apr. 2006. 17 pages.

[9] C. Fournet and M. Abadi. Hiding names: Private authentication in the applied pi calculus. In Proc. of Int. Symposium on Software Security (ISSS'02), volume 2609 of LNCS, pages 317-338, Tokyo, Japan, 2003. Springer.

[10] A. Fujioka, T. Okamoto, and K. Ohta. A practical secret voting scheme for large scale elections. In Proc. of $A d$ vances in Cryptology (Asiacrypt'92), volume 718 of LNCS, pages 244-251, Gold Coast, Queensland, Australia, 1992. Springer.

[11] M. Hirt and K. Sako. Ef£cient receipt-free voting based on homomorphic encryption. In Proc. of Advances in Cryptography (Eurocrypt'00), volume 1807 of LNCS, pages 539556, Bruges, Belgium, 2000. Springer.

[12] W.-S. Juang and C.-L. Lei. A secure and practical electronic voting scheme for real world environments. IEICE Transaction on Fundamentals of Electronics, Communications and Computer Science, 1:64-71, 1997.

[13] A. Juels, D. Catalano, and M. Jakobsson. Coercion-resistant electronic elections. In Proc. of Workshop on Privacy in the Electronic Society (WPES'05), Alexandria, USA, 2005. ACM Press.

[14] S. Kremer and M. D. Ryan. Analysis of an electronic voting protocol in the applied pi-calculus. In Proc. 14th European Symposium On Programming (ESOP'05), volume 3444 of LNCS, pages 186-200, Edinburgh, U.K., 2005. Springer.

[15] B. Lee, C. Boyd, E. Dawson, K. Kim, J. Yang, and S. Yoo. Providing receipt-freeness in mixnet-based voting protocols. In Proc. of Information Security and Cryptology (ICISC'03), volume 2971 of $L N C S$, pages 245-258, Seoul, Korea, 2004. Springer.

[16] T. Okamoto. An electronic voting scheme. In IFIP World Conference on IT Tools, pages 21-30, Canberra, Australia, 1996.

[17] T. Okamoto. Receipt-free electronic voting schemes for large scale elections. In Proc. 5th Int. Security Protocols Workshop, volume 1361 of LNCS, pages 25-35, Paris, France, 1997. Springer.

[18] S. Schneider and A. Sidiropoulos. CSP and anonymity. In Proc. 4th European Symposium On Research In Computer Security (ESORICS'96), volume 1146 of LNCS, pages 198218, Roma, Italy, 1996. Springer. 